\title{
Sparse Signal Recovery for Direction-of-Arrival Estimation Based on Source Signal Subspace
}

\author{
Bo Lin, Jiying Liu, Meihua Xie, and Jubo Zhu \\ College of Science, National University of Defense Technology, Changsha 410073, China \\ Correspondence should be addressed to Bo Lin; linbo@nudt.edu.cn
}

Received 10 January 2014; Accepted 28 March 2014; Published 30 April 2014

Academic Editor: Huijun Gao

Copyright ( 2014 Bo Lin et al. This is an open access article distributed under the Creative Commons Attribution License, which permits unrestricted use, distribution, and reproduction in any medium, provided the original work is properly cited.

\begin{abstract}
After establishing the sparse representation of the source signal subspace, we propose a new method to estimate the direction of arrival (DOA) by solving an $\ell_{1}$-norm minimization for sparse signal recovery of the source powers. Second-order cone programming is applied to reformulate this optimization problem, and it is solved effectively by employing the interior point method. Due to the keeping of the signal subspace and the discarding of the noise subspace, the proposed method is more robust to noise than many other sparsity-based methods. The real data tests and the numerical simulations demonstrate that the proposed method has improved accuracy and robustness to noise, and it is not sensitive to the knowledge about the number of sources. We discuss the computational cost of our method theoretically, and the experiment results verify the computational effectiveness.
\end{abstract}

\section{Introduction}

Sparse signal recovery (SSR) is an emerging field and has received considerable attention in recent years [1-4]. It has been applied widely in many fields, such as medical imaging $[5,6]$, wireless communication and telemonitoring $[7,8]$, and sensor networks $[9,10]$. In the framework of standard SSR (sSSR) with complex data, the mathematical model of measurement is

$$
\mathbf{y}=\Phi \mathbf{x}+\mathbf{n}
$$

where $\mathbf{y} \in \mathbb{C}^{M \times 1}$ is an available measurement vector, $\Phi \in$ $\mathbb{C}^{M \times N}$ is a known projection matrix with $M$ significantly smaller than $N, \mathbf{n}$ is a noise term, and $\mathbf{x} \in \mathbb{C}^{N \times 1}$ is the original signal vector to estimate. In order to ensure the uniqueness of the signal recovered from the underdetermined system (1), $\mathbf{x}$ should be $K$-sparse and $K \ll N$. Under the assumption that the estimation of $\mathbf{x}$ is sparest, the intuitive estimator is

$$
\mathbf{x}^{*}=\arg \min _{\mathbf{x} \in \mathbb{C}^{N \times 1}}\|\mathbf{x}\|_{0} \quad \text { s.t. }\|\mathbf{y}-\boldsymbol{\Phi} \mathbf{x}\|_{2} \leq \epsilon,
$$

where $\|\mathbf{x}\|_{0}=\#\left\{x_{n} \mid x_{n} \neq 0\right.$ and $\left.x_{n} \in \mathbf{x}\right\}$ denotes the number of the nonzero entries in $\mathbf{x}$ and $\epsilon^{2}$ is an appropriately upper bound on the noise power $\|\mathbf{n}\|_{2}^{2}$. As we know, however, it is NP-hard to solve this combinatorial optimization. Fortunately, some researches $[1,2]$ guarantee that if $\mathbf{x}$ is reasonably sparse and $\Phi$ satisfies some suitable conditions, the $\ell_{1}$-norm minimization (3) is equivalent to (2):

$$
\mathbf{x}^{*}=\arg \min _{\mathbf{x} \in \mathbb{C}^{N \times 1}}\|\mathbf{x}\|_{1} \quad \text { s.t. }\|\mathbf{y}-\boldsymbol{\Phi} \mathbf{x}\|_{2} \leq \epsilon,
$$

where $\|\mathbf{x}\|_{1}=\sum_{n=1}^{N}\left|x_{n}\right|$. This optimization (3) is a convex relaxation and can be solved by many algorithms.

Array processing is used widely in many applications, including electronic warfare and civil aviation. During the past years, besides the design and the improvement about the electronic of the array system [11, 12], a lot of mathematical methods have been proposed to improve the performance of array processing. Due to the spatial sparsity of the incident sources, the sSSR methodology (3) and its extension to the multiple measurement vector (MMV) model [13-15] are exploited to accomplish the direction-of-arrival (DOA) estimation based on the array processing [16-27]. Compared with the classical superresolution methods, such as MUSIC [28], these sparsity-based methods have many advantages, including requirement of only a few snapshots, dealing with the correlated sources, and better performance. For almost all of them, however, the measurement domain is either the array output or the array covariance matrix. In this paper, 
we propose a novel sparsity-based method for DOA estimation by exploiting the source signal subspace as the measurement domain. Firstly, we provide a method based on the sSSR methodology using the array covariance, which we refer to as sSSR-AC. On the basis of sSSR-AC, we present a sparse representation of the signal subspace and establish the sSSR model of the source powers, and finally a second-order cone programming (SOCP) [29] is applied to formulate this sSSR problem. The SOCP is solved by interior point technique [30]. We refer to this method as sSSR-SS. Because the sSSR is implemented in the signal subspace by discarding the noise part, sSSR-SS is more robust to noise than other sparsitybased methods which use the array output or array covariance as the measurement domain.

The rest of this paper is organized as follows. In Section 2, after presenting the sparse representation of the signal subspace, we formulate the DOA estimation as an sSSR problem. In Section 3, we transform this sSSR problem into an SOCP form and obtain its solution by employing the interior point method, and we analyze the computational cost of the proposed method. Finally, we carry out the real data tests and the numerical simulations in Section 4 and make the conclusions in Section 5.

The notations used in this paper are introduced as follows: $\mathbf{I}_{L}$ denotes the identity matrix with size $L \times L$, and, for simplicity, I stands for the identity matrix, of which the dimension can be determined from the context. $\mathbf{A} \otimes \mathbf{B}$ represents the Kronecker product of the matrices $\mathbf{A}$ and $\mathbf{B}$. $\operatorname{vec}(\mathbf{A})$ denotes the vectorization of matrix $\mathbf{A}$ by stacking its columns one by one into a single-column vector. $\mathbf{A}^{T}$ and $\mathbf{A}^{H}$ are the transpose and the conjugate transpose of $\mathbf{A}$, respectively. $\operatorname{Re}(\mathbf{A})$ and $\operatorname{Im}(\mathbf{A})$ denote the real and the image parts of $\mathbf{A}$.

\section{Sparse Signal Recovery for DOA Estimation}

Assume that $K$ far-field narrowband and motionless sources impinge on an $M$-sensor linear array from directions $\boldsymbol{\theta}=$ $\left[\theta_{1}, \theta_{2}, \ldots, \theta_{K}\right]^{T}$. The array output contaminated by noise is represented as

$$
\mathbf{y}(t)=\mathbf{A}(\boldsymbol{\theta}) \mathbf{s}(t)+\mathbf{n}(t), \quad t=1,2, \ldots, L,
$$

where $\mathbf{s}(t)=\left[s_{1}(t), s_{2}(t), \ldots, s_{K}(t)\right]^{T}$ is the spatial signal vector of the incident sources, $s_{k}(t)$ is the signal waveform of the $k$ th source, and $\mathbf{n}(t)$ is a zero-mean additive complex Gaussian white noise with the covariance matrix $\mathbf{R}_{\mathbf{n}}=\sigma^{2} \mathbf{I}$. The noise is uncorrelated with source signals. $L$ is the number of the snapshots. $\mathbf{A}(\boldsymbol{\theta})$ is the array manifold matrix and denoted as

$$
\mathbf{A}(\boldsymbol{\theta})=\left[\mathbf{a}\left(\theta_{1}\right), \mathbf{a}\left(\theta_{2}\right), \ldots, \mathbf{a}\left(\theta_{K}\right)\right]
$$

where $\mathbf{a}\left(\theta_{k}\right)=\left[e^{-j 2 \pi f_{0} \tau_{1, k}}, e^{-j 2 \pi f_{0} \tau_{2, k}}, \ldots, e^{-j 2 \pi f_{0} \tau_{M, k}}\right]^{T}$ is the array steering vector corresponding to the $k$ th sources, $f_{0}$ denotes the carrier frequency, and $\tau_{m, k}$, which is related to the arriving direction $\theta_{k}$, is the propagational time-delay of the $k$ th signal between the reference and the $m$ th sensor of the array. The covariance matrix of the array output is calculated by

$$
\mathbf{R}_{\mathbf{y}}=E\left\{\mathbf{y}(t) \mathbf{y}^{H}(t)\right\}=\mathbf{A R}_{\mathbf{s}} \mathbf{A}^{H}+\sigma^{2} \mathbf{I}
$$

where $\mathbf{R}_{\mathbf{s}}=E\left\{\mathbf{s}(t) \mathbf{s}^{H}(t)\right\}$ is the covariance matrix of the incident source signals.

2.1. $s S S R-A C$. Since the spatial sources are distributed sparsely, the potential bearing space of the incident sources can be discretized to form a spatial sampling grid $\Theta=$ $\left[\vartheta_{1}, \vartheta_{2}, \ldots, \vartheta_{N}\right]^{T}$ with $N \gg K$. For convenience of description, we assume $\boldsymbol{\theta} \subset \boldsymbol{\Theta}$. $\mathbf{x}(t)=\left[x_{1}(t), x_{2}(t), \ldots, x_{N}(t)\right]^{T}$ is defined as a zero-padded version of the signal vector $\mathbf{s}(t)$ from $\boldsymbol{\theta}$ to $\Theta$; that is, if there exists $\vartheta_{n}=\theta_{k}$, then $x_{n}(t)=s_{k}(t)$; and otherwise, $x_{n}(t)=0$. Therefore, $\mathbf{x}(t)$ is a $K$-sparse vector. Correspondingly, $\boldsymbol{\Phi}(\boldsymbol{\Theta}) \in \mathbb{C}^{M \times N}$ is an extension of the array manifold matrix $\mathbf{A}(\boldsymbol{\theta})$; that is

$$
\boldsymbol{\Phi}(\boldsymbol{\Theta})=\left[\mathbf{a}\left(\vartheta_{1}\right), \mathbf{a}\left(\vartheta_{2}\right), \ldots, \mathbf{a}\left(\vartheta_{N}\right)\right] .
$$

Then the array output (4) is represented as a sparse linear combination of $\Phi(\Theta)$

$$
\mathbf{y}(t)=\boldsymbol{\Phi}(\boldsymbol{\Theta}) \mathbf{x}(t)+\mathbf{n}(t), \quad t=1,2, \ldots, L,
$$

and thus the covariance matrix (6) can be rewritten as

$$
\mathbf{R}_{\mathbf{y}}=\boldsymbol{\Phi} \mathbf{R}_{\mathbf{x}} \boldsymbol{\Phi}^{H}+\sigma^{2} \mathbf{I}
$$

with $\mathbf{R}_{\mathbf{x}}=E\left\{\mathbf{x}(t) \mathbf{x}^{H}(t)\right\}$. Under the assumption that the sources are spatially uncorrelated, we have $\mathbf{R}_{\mathbf{x}}=\operatorname{diag}\left(\sigma_{1}^{2}\right.$, $\sigma_{2}^{2}, \ldots, \sigma_{N}^{2}$ ), and $\sigma_{n}^{2}$ is the power of the $n$th source signal. Thus, (9) can be reformulated as

$$
\mathbf{r}=\Psi \mathbf{p}+\sigma^{2} \mathbf{I}_{\mathbf{v}}
$$

where $\mathbf{r}=\operatorname{vec}\left(\mathbf{R}_{\mathbf{y}}\right) \in \mathbb{C}^{M^{2} \times 1}, \mathbf{I}_{\mathbf{v}}=\operatorname{vec}(\mathbf{I}) \in \mathbb{R}^{M^{2} \times 1}, \Psi=$ $\left[\mathbf{b}\left(\vartheta_{1}\right), \mathbf{b}\left(\vartheta_{2}\right), \ldots, \mathbf{b}\left(\vartheta_{N}\right)\right] \in \mathbb{C}^{M^{2} \times N}$ with $\mathbf{b}\left(\vartheta_{n}\right)=\mathbf{a}^{H}\left(\vartheta_{n}\right) \otimes$ $\mathbf{a}\left(\vartheta_{n}\right) \in \mathbb{C}^{M^{2} \times 1}$, and $\mathbf{p}=\left[\sigma_{1}^{2}, \sigma_{2}^{2}, \ldots, \sigma_{N}^{2}\right]^{T}$. Actually, $\sigma_{n}^{2}=0$ if $x_{n}(t)=0$ for $n \in[1,2, \ldots, N]$. Therefore, $\mathbf{p}$ is also a $K$ sparse vector, and the DOA estimation is transformed into a problem of sparse signal recovery of $\mathbf{p}$ with the array out $\mathbf{y}(t)$ and the overcomplete basis $\Phi$ available.

Since $\mathbf{p}$ is $K$-sparse and $K \ll N$, we can solve the inverse problem of sparse signal recovery using $\ell_{1}$-norm minimization, and the estimator is

$$
\mathbf{p}^{*}=\arg \min _{\mathbf{p} \in \mathbb{R}^{N \times 1}}\|\mathbf{p}\|_{1} \quad \text { s.t. }\|\mathbf{r}-\Psi \mathbf{p}\|_{2} \leq \beta,
$$

where $\beta$ is a positive parameter and we assume that a good choice of $\beta$ is made. After $\mathbf{p}$ is solved from (11), the DOA estimation of the true sources is obtained according to the support or the peaks of $\mathbf{p}^{*}$. We will adopt an SOCP described in Section 3 to solve (11). We call the estimator (11) as sSSRAC. According to the description as above, it is obvious that sSSR-AC does not require a priori information about the number of the true sources. 
Mathematically, we can obtain the DOAs estimation by solving

$$
\begin{array}{ll}
\min & \|\mathbf{x}(t)\|_{1} \\
\text { s.t. } & \|\mathbf{y}(t)-\mathbf{\Phi} \mathbf{x}(t)\|_{2} \leq \varepsilon
\end{array}
$$

based on the single snapshot model (8). However, the estimator (11), which uses the multiple snapshots in synergy, will be more robust to noise and achieve better accuracy. The main drawback of the estimator (11) is that the size and the computational cost of the optimization increase quadratically with the number of the sensors $M$.

2.2. sSSR-SS. In order to overcome the computational drawback of sSSR-AC, we introduce the subspace method. We decompose the array covariance matrix $\mathbf{R}_{\mathbf{y}}$ to obtain the source signal subspace. If the dimension of the signal subspace is smaller than the number of the sensors, we use this signal subspace to replace the array covariance. This technique will reduce the dimension and computational cost. Moreover, the subspace decomposition will improve the robustness to noise by keeping the signal subspace and discarding the noise one.

Take the eigenvalue decomposition (EVD) of $\mathbf{R}_{\mathbf{y}}$ :

$$
\mathbf{R}_{\mathbf{y}}=\mathbf{U} \boldsymbol{\Sigma} \mathbf{U}^{H}
$$

then the first $K$ columns of the matrix $\mathbf{U}$, corresponding to the $K$ largest eigenvalues of $\mathbf{R}_{\mathbf{y}}$, are the basis of the signal subspace, and $K$ is the dimension of this subspace. In the application of array signal processing, the number $M$ of the sensors is always not very large so that the computational workload of this EVD is quite small.

Here we do not use the signal subspace directly by taking the linear combination of the first $K$ eigenvectors. Instead of that, we consider the parallel alliance of these eigenvectors. By Setting $\mathbf{D}=\left[\begin{array}{ll}\mathbf{I}_{K} & \mathbf{0}\end{array}\right]^{T} \in \mathbb{R}^{M \times K}$, where $\mathbf{0}$ is a zero matrix with dimension $K \times(M-K)$, we get the parallel alliance of these bases:

$$
\mathrm{Z}=\mathbf{U} \boldsymbol{\Sigma} \mathbf{D}=\mathbf{R}_{\mathbf{y}} \mathbf{U D} .
$$

Now we multiply two sides of (9) simultaneously by UD to obtain

$$
\mathbf{Z}=\boldsymbol{\Phi} \mathbf{R}_{\mathbf{x}}\left(\boldsymbol{\Phi}^{H} \mathbf{U D}\right)+\sigma^{2} \mathbf{U D}
$$

where $\mathbf{Z} \in \mathbb{C}^{M \times K}$. Let $\boldsymbol{\Omega}=\mathbf{D}^{H} \mathbf{U}^{H} \boldsymbol{\Phi}$ and write as $\boldsymbol{\Omega}=$ $\left[\boldsymbol{\omega}_{\cdot, 1}, \boldsymbol{\omega}_{\cdot, 2}, \ldots, \boldsymbol{\omega}_{\cdot, N}\right] \in \mathbb{C}^{K \times N}$, where $\boldsymbol{\omega}_{\cdot, n}$ denotes the $n$th column of $\Omega$; then similarly to (10), the vectorization version of the signal subspace model (15) is represented as

$$
\mathbf{z}=\boldsymbol{\Xi} \mathbf{p}+\sigma^{2} \mathbf{e}
$$

where $\mathbf{z}=\operatorname{vec}(\mathbf{Z}) \in \mathbb{C}^{M K \times 1}, \mathbf{e}=\operatorname{vec}(\mathbf{U D}) \in \mathbb{C}^{M K \times 1}, \boldsymbol{\Xi}=$ $\left[\boldsymbol{\xi}_{\cdot, 1}, \boldsymbol{\xi}_{\cdot, 2}, \ldots, \boldsymbol{\xi}_{\cdot, N}\right] \in \mathbb{C}^{M K \times N}$ with $\boldsymbol{\xi}_{\cdot, n}=\boldsymbol{\omega}_{\cdot, n}^{H} \otimes \mathbf{a}\left(\vartheta_{n}\right) \in \mathbb{C}^{M K \times 1}$, and $\mathbf{p}=\left[\sigma_{1}^{2}, \sigma_{2}^{2}, \ldots, \sigma_{N}^{2}\right]^{T}$. Therefore, the estimator of $\mathbf{p}$ is

$$
\mathbf{p}_{\text {ss }}^{*}=\arg \min \|\mathbf{p}\|_{1} \quad \text { s.t. }\|\mathbf{z}-\boldsymbol{\Xi} \mathbf{p}\|_{2} \leq \delta,
$$

where $\delta$ is a positive parameter and we assume that a good choice of $\delta$ is also made. The problem (17) will be manipulated into the SOCP form and then solved by interior point method. We refer to this method as sSSR-SS. Compared with (11), it is obvious that when $K<M$, the computational complexity of sSSR-SS will be lower than that of sSSR-AC.

\section{SOCP Form of Sparse Signal Recovery}

In the sparse signal recovery (3), the objection $\|\mathbf{x}\|_{1}=$ $\sum_{n=1}^{N} \sqrt{\operatorname{Re}\left(x_{i}\right)^{2}+\operatorname{Im}\left(x_{i}\right)^{2}}$ is neither linear nor quadratic about the real and the imaginary components of $\mathbf{x}$. To deal with this problem, we transform (3) into an SOCP problem, the general form of which is

$$
\begin{array}{ll}
\min & \mathbf{f}^{T} \mathbf{t} \\
\text { s.t. } & \left\|\mathbf{A}_{i} \mathbf{t}+\mathbf{b}_{i}\right\|_{2} \leq \mathbf{c}_{i}^{T} \mathbf{t}+d_{i} \quad i=1,2, \ldots, h, \\
& \mathbf{F y}=\mathbf{g},
\end{array}
$$

where $\mathbf{t} \in \mathbb{R}^{m}$ is the optimization variable, and the known parameters are $\mathbf{f} \in \mathbb{R}^{m}, \mathbf{A}_{i} \in \mathbb{R}^{\left(m_{i}-1\right) \times m}, \mathbf{b}_{i} \in \mathbb{R}^{m_{i}-1}, \mathbf{c}_{i} \in \mathbb{R}^{m}$, $d_{i} \in \mathbb{R}, \mathbf{F} \in \mathbb{R}^{r \times m}$, and $\mathbf{g} \in \mathbb{R}^{r}$.

The second-order cone of dimension $n$ is defined as

$$
\operatorname{SOC}^{n}=\left\{\left[\begin{array}{l}
y \\
\mathbf{x}
\end{array}\right] \mid y \in \mathbb{R}, \mathbf{x} \in \mathbb{R}^{n-1},\|\mathbf{x}\|_{2} \leq y\right\},
$$

and zero cone of dimension $r$ is

$$
\mathbf{0}^{r}=\left\{\mathbf{x} \mid \mathbf{x} \in \mathbb{R}^{r}, \mathbf{x}=\mathbf{0}\right\},
$$

so the constraints of (18) are equivalent to

$$
\begin{gathered}
{\left[\begin{array}{c}
\mathbf{c}_{i}^{T} \mathbf{t}+d_{i} \\
\mathbf{A}_{i} \mathbf{t}+\mathbf{b}_{i}
\end{array}\right] \in \mathrm{SOC}^{m_{i}},} \\
\mathbf{F y}-\mathbf{g} \in \mathbf{0}^{r} .
\end{gathered}
$$

Thus, we call the optimization (18) as SOCP. It is a convex optimization, since the objection is a convex function and the feasible region is a convex set. Actually, SOCP is a general framework for several convex optimization problems. For example, when $\mathbf{c}_{i}=\mathbf{0}$ for all $i$, the SOCP (18) is reduced to a quadratically constrained linear program (QCLP) problem.

By defining an auxiliary variable $\mathbf{v}=\mathbf{y}-\boldsymbol{\Phi} \mathbf{x}$ and a new matrix

$$
\widetilde{\boldsymbol{\Phi}}=\left[\begin{array}{cc}
\operatorname{Re}(\boldsymbol{\Phi}) & \operatorname{Im}(\boldsymbol{\Phi}) \\
-\operatorname{Im}(\boldsymbol{\Phi}) & \operatorname{Re}(\boldsymbol{\Phi})
\end{array}\right]
$$

we can represent the sparse signal recovery (3) as an SOCP, exactly QCLP, as follows:

$$
\begin{array}{ll}
\min & \mathbf{c}^{T} \mathbf{t} \\
\text { s.t. } & \left\|\left[\begin{array}{l}
\operatorname{Re}\left(x_{i}\right) \\
\operatorname{Im}\left(x_{i}\right)
\end{array}\right]\right\|_{2} \leq u_{i}, \quad i=1,2, \ldots, N, \\
& \left\|\left[\begin{array}{l}
\operatorname{Re}(\mathbf{v}) \\
\operatorname{Im}(\mathbf{v})
\end{array}\right]\right\|_{2} \leq \epsilon, \\
& \mathbf{B t}=\mathbf{d},
\end{array}
$$


where $\mathbf{c}=\left[1,0,0,1,0,0, \ldots, 1,0,0, \mathbf{0}_{2 M+1}^{T}\right] \in \mathbb{R}^{3 N+2 M+1}$, $\mathbf{0}_{2 M+1}$ is a zero-column vector with dimension $2 M+1$, $\operatorname{Re}(\mathbf{v})=\left[\operatorname{Re}\left(v_{1}\right), \operatorname{Re}\left(v_{2}\right), \ldots, \operatorname{Re}\left(v_{M}\right)\right]^{T}, \operatorname{Im}(\mathbf{v})=\left[\operatorname{Im}\left(v_{1}\right)\right.$, $\left.\operatorname{Im}\left(v_{2}\right), \ldots, \operatorname{Im}\left(v_{M}\right)\right]^{T}, \mathbf{d}=\left[\operatorname{Re}(\mathbf{y})^{T}, \operatorname{Im}(\mathbf{y})^{T}, \epsilon\right]^{T}$, and the matrix $\mathbf{B}$ is expressed as

$$
\mathbf{B}=\left[\begin{array}{c|c}
\mathbf{B}_{1} & \mathbf{B}_{2} \\
\hline \mathbf{B}_{3} & \mathbf{0}
\end{array}\right] \in \mathbb{R}^{(2 M+1) \times(3 N+1+2 M)},
$$

where $\mathbf{B}_{1}=\left[\mathbf{0}, \widetilde{\boldsymbol{\Phi}}_{\cdot, 1}, \widetilde{\boldsymbol{\Phi}}_{\cdot, N+1}, \mathbf{0}, \widetilde{\boldsymbol{\Phi}}_{\cdot, 2}, \widetilde{\boldsymbol{\Phi}}_{\cdot, N+2}, \ldots, \mathbf{0}, \widetilde{\boldsymbol{\Phi}}_{\cdot, N}\right.$, $\left.\widetilde{\boldsymbol{\Phi}}_{\cdot, N+N}, \mathbf{0}\right] \in \mathbb{R}^{2 M \times(3 N+1)}, \widetilde{\boldsymbol{\Phi}}_{\cdot, i}$ means the $i$ th column of the matrix $\widetilde{\boldsymbol{\Phi}}, \mathbf{B}_{2}=\mathbf{I}_{2 M}$, and $\mathbf{B}_{3}=[0,0, \ldots, 0,1] \in \mathbb{R}^{1 \times(3 N+1)}$. The optimization variable is $\mathbf{t}=\left[u_{1}, \operatorname{Re}\left(x_{1}\right), \operatorname{Im}\left(x_{1}\right), u_{2}\right.$, $\operatorname{Re}\left(x_{2}\right), \operatorname{Im}\left(x_{2}\right), \ldots, u_{N}, \operatorname{Re}\left(x_{N}\right), \operatorname{Im}\left(x_{N}\right), \epsilon, \operatorname{Re}\left(v_{1}\right), \operatorname{Re}\left(v_{2}\right), \ldots$, $\left.\operatorname{Re}\left(v_{M}\right), \operatorname{Im}\left(v_{1}\right), \operatorname{Im}\left(v_{2}\right), \ldots, \operatorname{Im}\left(v_{M}\right)\right]^{T}$, which in- cludes the real and the image parts of the sparse signal $\mathbf{x}$ that we pursue.

We employ the package called SeDuMi [31] to obtain the numerical solution of the SOCP (23) by using the interior point method. The computational complexity of the interior point method depends on the number of iterations and the computational workload per iteration. For (23), the cost of each iteration is $O\left((3 N+2 M+1)^{3}\right)$. However, the number of iterations is worst-case bound above by $O(\sqrt{N+1})$, which is independent of the size $M$ of the array. Therefore, the computational complexity of the SOCP for (17) is about $\left(O(3 N+2 M K+1)^{3}\right)$ and that for (11) is about $O\left(\left(3 N+2 M^{2}+\right.\right.$ $1)^{3}$ ). When $K<M$, the latter is higher than the former.

\section{Experiments}

In this section, we carry out several experiments to investigate the performance of our sSSR-AC and sSSR-SS. Firstly, we compare the spectra of them with those of $\ell_{1}$-SVD [16], OGSBI-SVD [25], and MUSIC [28] both in real data tests and numerical simulations, respectively. Then, we analyze the statistical performance of them by comparing the root mean square error (RMSE) of DOA estimation in various scenarios. Here, the RMSE is defined as

$$
\mathrm{RMSE}=\sqrt{\frac{\sum_{m c=1}^{M C} \sum_{k=1}^{K}\left(\widehat{\theta}_{k}^{(m c)}-\theta_{k}^{(m c)}\right)^{2}}{K \cdot M C}},
$$

where $M C$ is the total number of Monte Carlo trials, $\theta_{k}^{(m c)}$ is the true direction of the $k$ th source in the $m c t h$ trial, and $\widehat{\theta}_{k}^{(m c)}$ is the estimation of $\theta_{k}^{(m c)}$.

In the following experiments, the uniform linear array (ULA) is taken into account. The number of the sensors is $M=8$, and the intersensor spacing is $d=0.07 \mathrm{~m}$. The incident sources are complex sinusoid signals with carrier frequency $f_{0}=2.143 \mathrm{GHz}$, so that the element spacing of the ULA is half the wavelength of the source signals. The sources are spatially uncorrelated. The uniform spatial sampling grid is equipped for $\ell_{1}$-SVD, MUSIC, and our methods. The grid interval is $\Delta \vartheta_{1}=0.1^{\circ}$. OGSBI-SVD algorithm is taken as one of the representatives of the methods based on off-grid model, and it is designed with grid interval $\Delta \vartheta_{2}=2^{\circ}$ for accurate yet fast DOA estimation (see [25] for details). The number of the sources is assumed to be known. The number of the snapshots is $L=256$.

4.1. Comparison of Spatial Spectra. Firstly we illustrate the practical validity of our methods by implementing the real data tests in the narrowband scenario. The real measurement data was collected by an antenna ULA consisting of 8 sensors for $S \backslash C$ waveband signals in a microwave black room. The sampling frequency was $500 \mathrm{Msps}$. The power of noise was unknown yet as low as possible. Before DOA estimation, the array output has been pretreated with phase correction and amplitude correction.

In Figure 1, we compare the spatial spectra of our methods sSSR-AC and sSSR-SS with those of $\ell_{1}$-SVD, OGSBI-SVD, and MUSIC. In Figure 1(a), the true directions of two sources are $-6.25^{\circ}$ and $7.05^{\circ}$. Our methods can distinguish the two sources as well as $\ell_{1}$-SVD and have higher resolution than MUSIC. In Figure 1(b), two sources come from $-6.25^{\circ}$ and $0^{\circ}$, and the angular separation between them is smaller than that in Figure 1(a). At this moment, our methods and $\ell_{1}$ SVD are still able to resolve the two sources clearly, while the peaks of MUSIC are not so distinct. Both in Figures 1(a) and 1(b), the spectrum peaks of OGSBI-SVD are quite indistinct, and maybe the reason is that the performance of OGSBI-SVD is not so good in the scenario of high SNR (signal-to-noise ratio), and the simulation results in Figure 2 illustrate this speculation, where the spectra of OGSBI-SVD are conspicuous in low SNR.

We set the SNR to $-5 \mathrm{~dB}$ and then the spatial spectra of the various methods are shown in Figure 2. The true directions of two sources are $\left[-5^{\circ}, 5^{\circ}\right]$ in Figure $2(\mathrm{a})$ and $\left[-5^{\circ}, 0^{\circ}\right]$ in Figure 2(b), respectively. As we can see, the peaks of sSSR-SS are consistently sharp as well as those in Figures 1(a) and 1(b). The results demonstrate that even though the SNR is low $(-5 \mathrm{~dB})$ and the source separation is small $\left(5^{\circ}\right)$, sSSR-SS has an excellent performance of DOA estimation consistently. Although the spectra of sSSR-AC show some false peaks in low SNR as shown in Figure 2, it has high accuracy for DOA estimation when the number of the true sources is known exactly (see Table 1).

Because of the implementation of SVD, we find that SSSRSS needs the knowledge of the number of sources. However, we illustrate that our SSSR-SS is not sensitive to the mistake of the source number. By using the real data used in Figure 1(a), we plot the spectra of sSSR-SS in Figure 3 for the assumed numbers of the sources, $K=1,3,5,7$, respectively. It is obvious that the variation among these spectra is very small. This result demonstrates the low sensitivity of sSSR-SS to the assumed number of the sources.

For the scenario of multiple sources, there is an attractive question that how many sources at most we can resolve by using our methods. Similarly to the $\ell_{1}$-SVD, our methods cannot separate $M$ sources simultaneously. However, our methods can resolve $M-1$ sources if the angular separation is not too small. The result shown in Figure 4 demonstrates this fact. In Figure 4 , the sources impinge on the array from $-60^{\circ}$, $-40^{\circ},-20^{\circ}, 0^{\circ}, 20^{\circ}, 40^{\circ}$, and $60^{\circ}$. The number of the sources is 7 , and the number of the sensors is $M=8$. The SNR is $-5 \mathrm{~dB}$. 


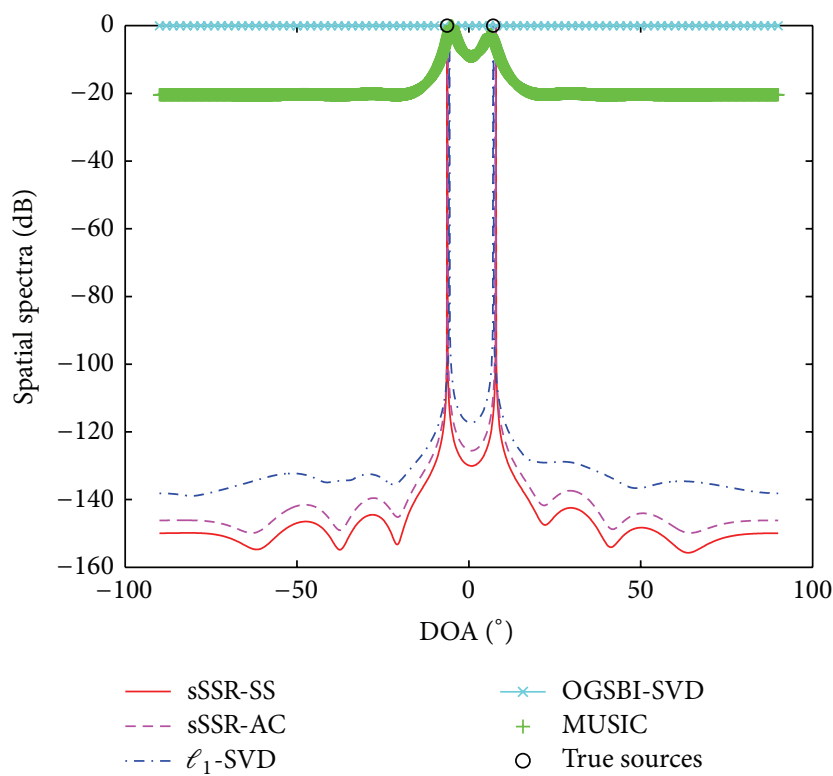

(a)

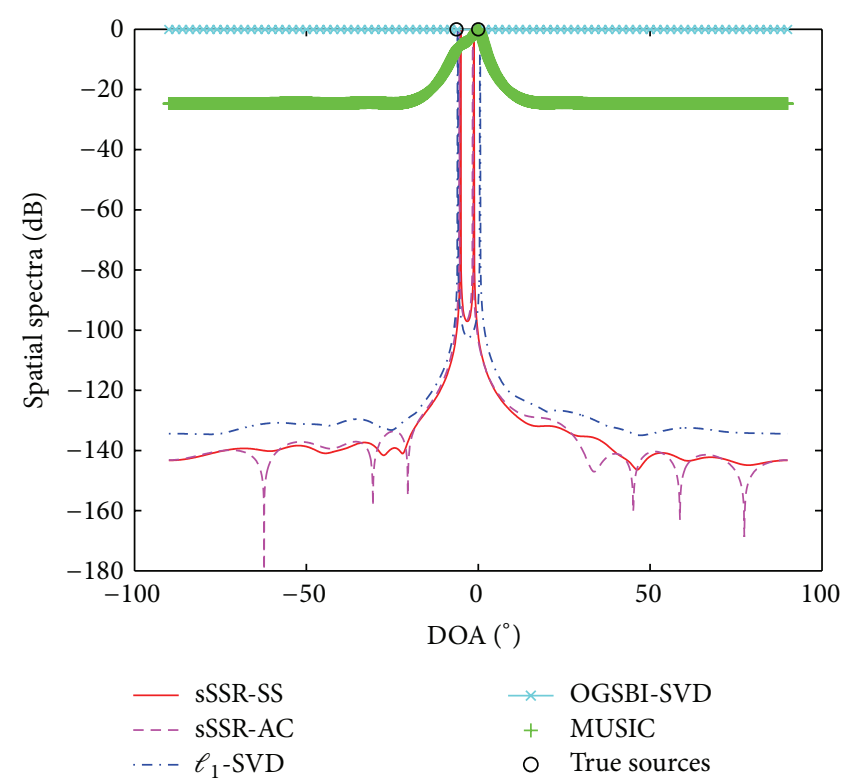

(b)

Figure 1: Spatial spectra of $\ell_{1}$-SVD, OGSBI-SVD, MUSIC, sSSR-AC, and sSSR-SS in real data tests for (a) sources from $\left[-6.25^{\circ}, 7.05^{\circ}\right]$ and (b) sources from $\left[-6.25^{\circ}, 0^{\circ}\right]$.

TABLE 1: The RMSE of DOA estimation in real data tests.

\begin{tabular}{lcccccc}
\hline & $0^{\circ}$ & $-6.25^{\circ}$ & $7.05^{\circ}$ & {$\left[-6.25^{\circ}, 0^{\circ}\right]$} & {$\left[0^{\circ}, 7.05^{\circ}\right]$} & {$\left[-6.25^{\circ}, 7.05^{\circ}\right]$} \\
\hline sSSR-SS & 0.1423 & 0.1194 & 0.0605 & 1.5447 & 1.0955 & 0.5803 \\
sSSR-AC & 0.1423 & 0.1194 & 0.0605 & 1.8943 & 0.2457 & 0.6024 \\
$\ell_{1}$-SVD & 0.1423 & 0.1197 & 0.0605 & 0.5746 & 1.2388 & 0.5307 \\
OGSBI-SVD & 0.7579 & 0.8815 & 0.5326 & 14.2306 & 11.4163 & 1.9361 \\
MUSIC & 0.1423 & 0.1197 & 0.0605 & 8.1617 & 6.8539 & 0.9076 \\
\hline
\end{tabular}

In this simulation, sSSR-SS and sSSR-AC have sharp peaks at the true directions as well as $\ell_{1}$-SVD.

The comparison results in the above experiments, especially in the real data tests, imply that our methods sSSR$\mathrm{AC}$ and sSSR-SS are effective for DOA estimation in the real application. According to the spatial spectra, we can suggest visually that they have some advantages, including that they have better resolution and surpass many other classical methods, the performance of them does not depend on the accurate estimation of the source number, and they can estimate and separate at most $M-1$ sources where $M$ is the number of the sensors. Particularly, sSSR-SS is quite robust to noise and has good performance in low SNR. All of the visual judgements will be further verified by the following experiments about the statistical performance.

4.2. Comparison of RMSE. In this section, we compare the RMSE of our methods to those of $\ell_{1}$-SVD, OGSBI-SVD, and MUSIC. For each scenario, 500 independent trials are carried out to calculate the RMSE.

We present the RMSE of sSSR-SS, sSSR-AC, and other compared methods using the real measurement data in
Table 1. We observe that, for scenario of single source or large angular separation, the accuracy of sSSR-SS and sSSR-AC is similar to that of $\ell_{1}$-SVD and MUSIC, while for scenario of small angular separation, the accuracy of our methods is slightly lower than that of $\ell_{1}-S V D$, yet much higher than that of MUSIC. Similarly to the result shown in Figure 1, the data in Table 1 demonstrates that our methods always surpass the OGSBI-SVD in all scenarios under consideration. The comparison results presented in Table 1 indicate that sSSR-SS and sSSR-AC have excellent performance and good resolution of DOA estimation in the real application.

We plot the RMSE curves of DOA estimation versus SNR in Figure 5(a). The SNR varies from $-12 \mathrm{~dB}$ to $0 \mathrm{~dB}$ with steplength $2 \mathrm{~dB}$. CRLB in Figure 5(a) denotes the Cramer-Rao lower bound (CRLB) of DOA estimation. We assume that two sources come from $-5^{\circ}+\Delta \theta$ and $5^{\circ}+\Delta \theta$, respectively, with $\Delta \theta$ produced uniformly and randomly within spatial interval $\left[-1^{\circ}, 1^{\circ}\right]$. Thus, the source directions are off-grid and the angular separation is always $10^{\circ}$ in each trial. Figure 5(a) reveals that the RMSE curve of sSSR-SS is closest to CRLB. sSSR-AC almost performs better than $\ell_{1}$-SVD and OGSBISVD all along, and it significantly outperforms MUSIC when 


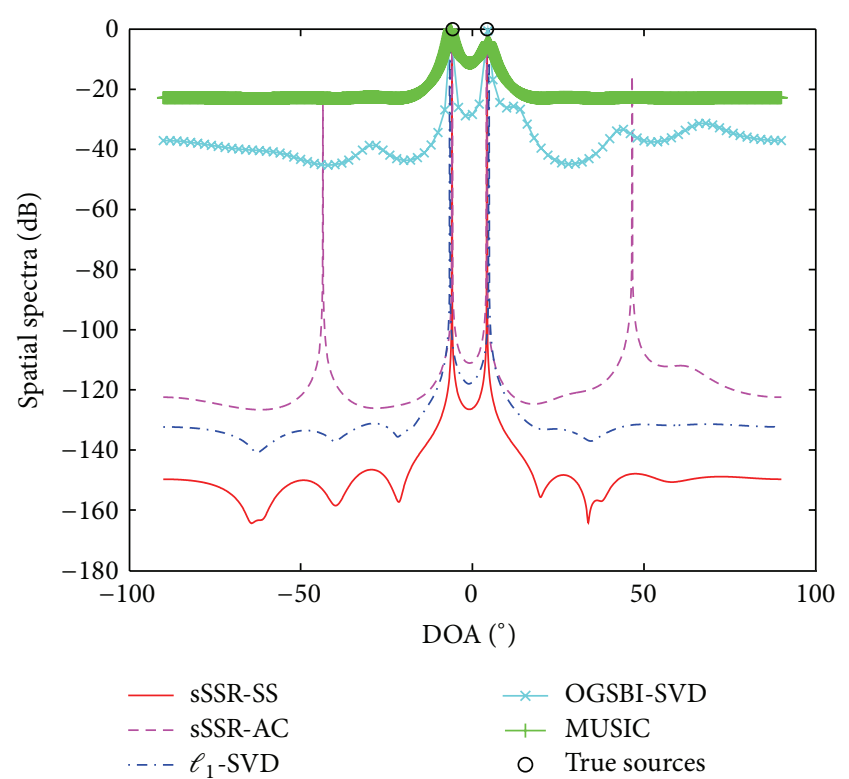

(a)

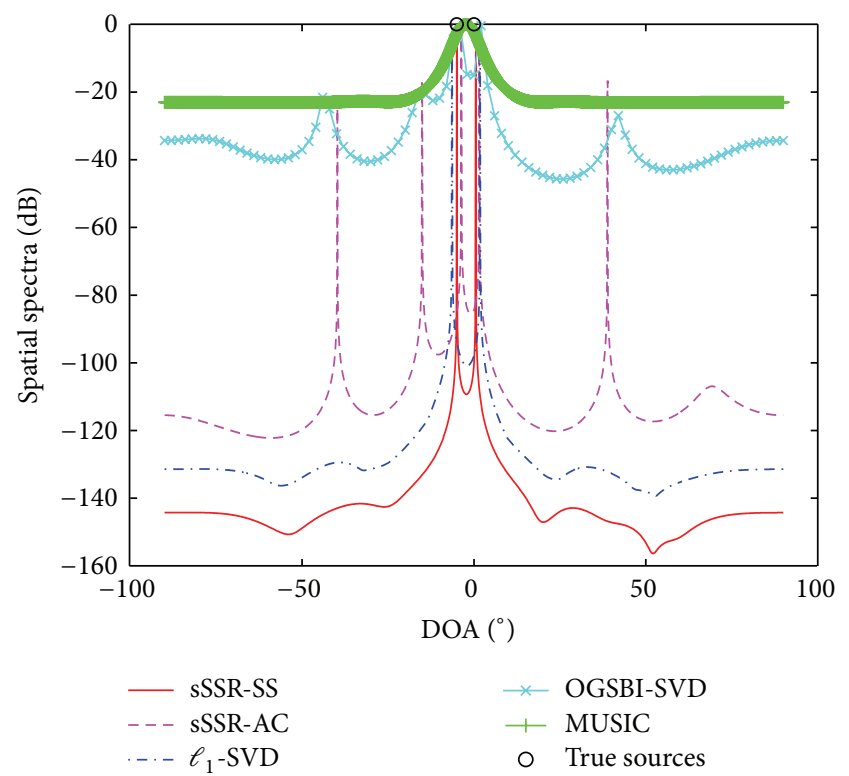

(b)

FIGURE 2: Spatial spectra of $\ell_{1}$-SVD, OGSBI-SVD, MUSIC, sSSR$A C$, and sSSR-SS in numerical simulations for (a) sources from $\left[-5^{\circ}, 5^{\circ}\right]$ and $(\mathrm{b})$ sources from $\left[-5^{\circ}, 0^{\circ}\right] . \mathrm{SNR}=-5 \mathrm{~dB}$.

the SNR is lower than $-6 \mathrm{~dB}$. The results shown in Figure 5(a) confirm that our methods surpass other methods and have remarkable robustness to noise.

In the next experiment, we examine the performance of our methods with respect to the angular separation between two sources. Consider the directions of two sources are $-\theta+$ $\Delta \theta$ and $\theta+\Delta \theta$ with $\Delta \theta$ produced uniformly and randomly within $\left[-1^{\circ}, 1^{\circ}\right]$; then the angular separation is $2 \theta$. We set $\theta=2,3, \ldots, 30$ and SNR $=-5 \mathrm{~dB}$. The curves of RMSE versus angular separation are plotted in Figure 5(b). The curves indicate that sSSR-SS and sSSR-AC outperform other

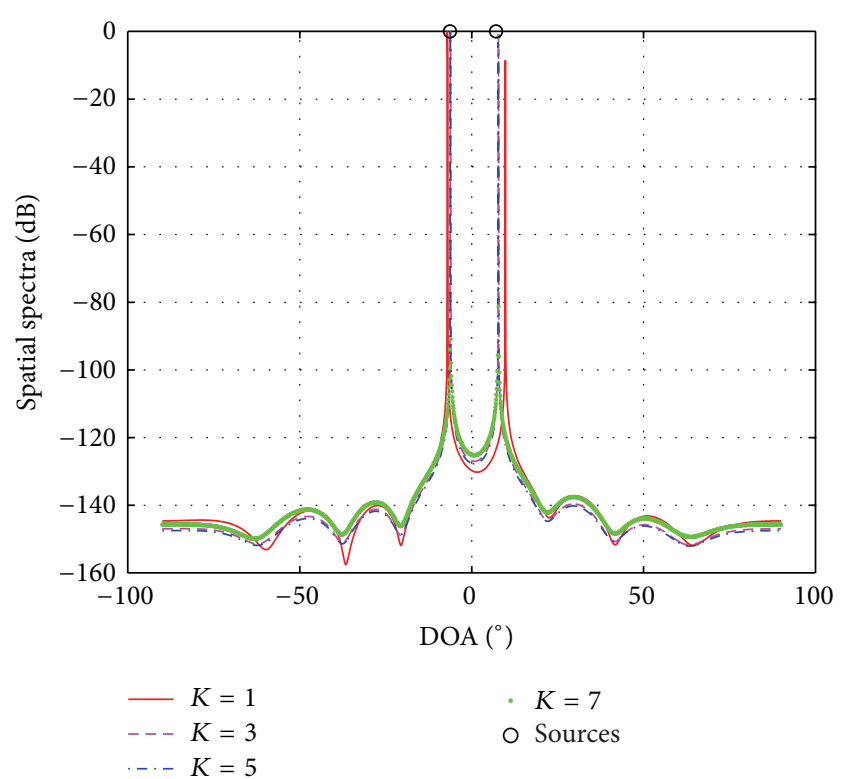

FIGURE 3: Spatial spectra of sSSR-SS in real data tests for the assumed number of the sources $K=1,3,5,7$, while the correct number of the sources is $K=2$.

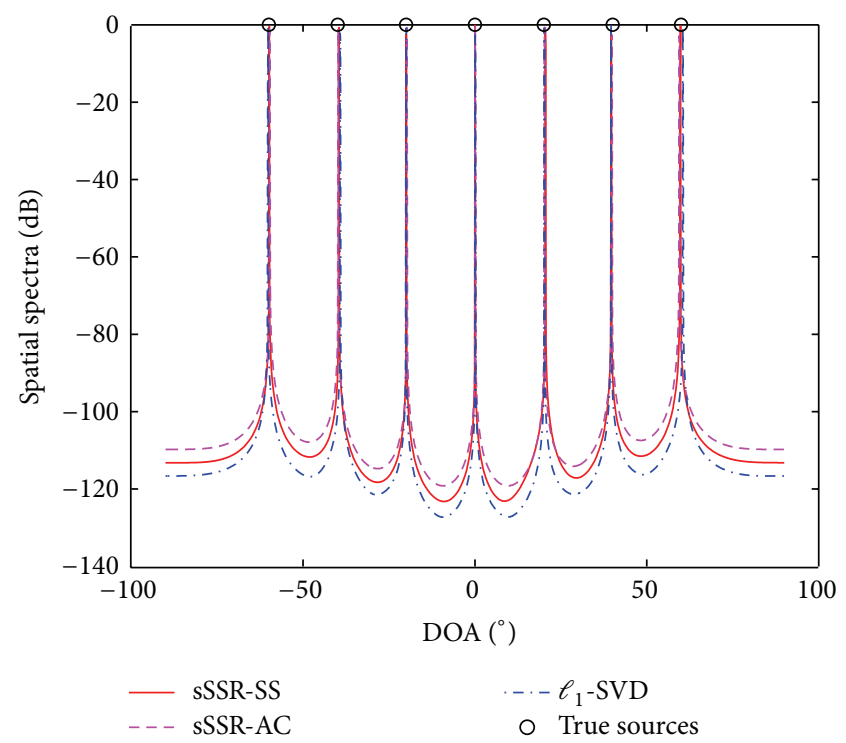

FIGURE 4: Spatial spectra for $M-1$ sources with SNR $=-5 \mathrm{~dB}$.

methods when the angular separation is smaller than $12^{\circ}$, while they perform as well as others in other scenarios under consideration. This result implies that our methods have the best capability for superresolution among these compared methods.

Finally we close this section by comparing the computational complexity of the various methods with respect to the number of the sensors $M$. The directions of two sources are set as in Figure 5(a). The SNR is set to $0 \mathrm{~dB}$, and the number of the sensors varies from 8 to 20 with step-length 2 . Figure 6 shows the average running time of the various methods calculated over 500 independent trials. The running 


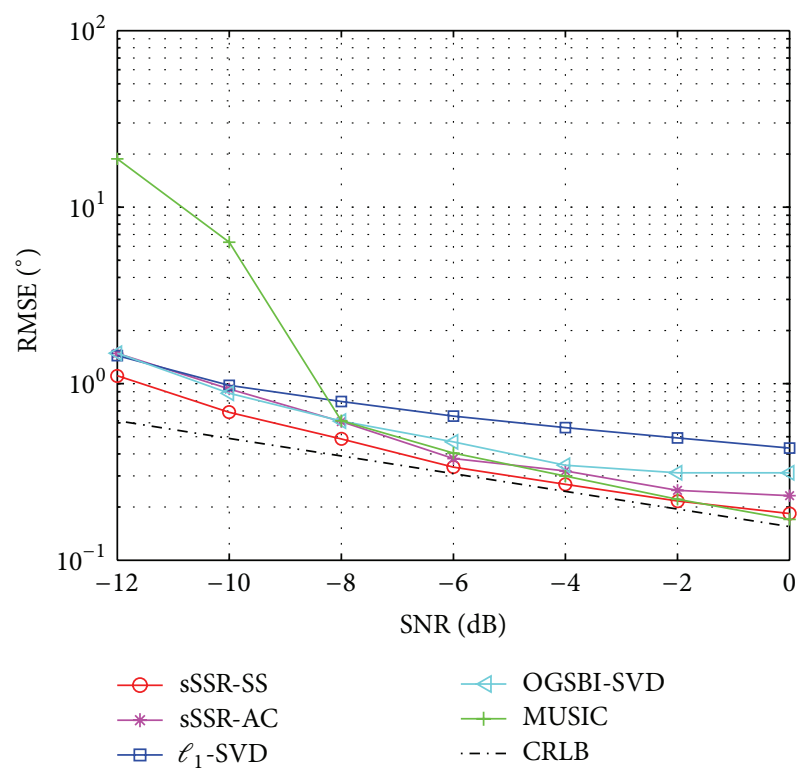

(a)

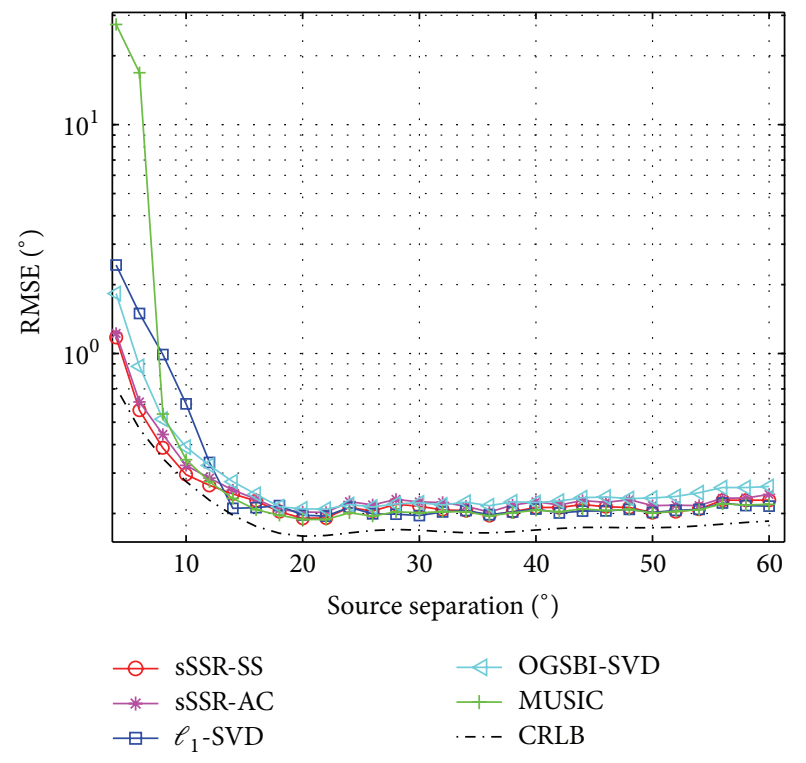

(b)

FIGURE 5: RMSE of $\ell_{1}$-SVD, OGSBI-SVD, MUSIC, sSSR-AC, and sSSR-SS with respect to (a) SNR with source separation $10^{\circ}$ fixed and (b) source separation with $\mathrm{SNR}=0 \mathrm{~dB}$ fixed.

time of sSSR-SS contains the cost of the implementation of SVD. The experiment is carried out in MATLAB v.7.8.0 on a PC with a Window XP system and a $4 \mathrm{GHz}$ CPU. The curve of sSSR-AC is a quadratic curve about $M$, and this fact verifies the theoretical analysis about the computational cost of sSSR-AC in Section 2.1. In addition, The curves of the average running time presented in Figure 6 illustrate that our sSSR-SS is faster than $\ell_{1}$-SVD and sSSR-AC, and this result confirms that the subspace decomposition technique is able to reduce the cost of sSSR-AC indeed. Although OGSBISVD and MUSIC run faster than sSSR-SS, the benefit of our

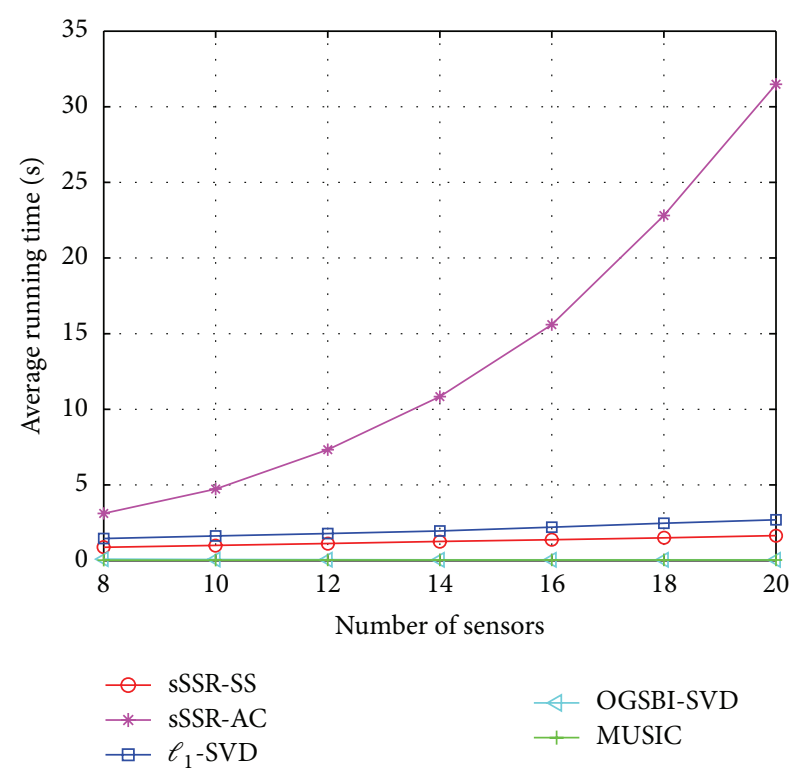

FIGURE 6: The average running time of $\ell_{1}$-SVD, OGSBI-SVD, MUSIC, sSSR-AC, and sSSR-SS with respect to the number of the sensors $M$.

methods is the higher accuracy of DOA estimation, and this fact has been illustrated by the above real data tests and the numerical simulations.

The comparison about the statistical performance of the various methods further confirms the advantages of our methods. Particularly, sSSR-SS based on the signal subspace reduces the computational cost and has superior robustness to noise.

\section{Conclusion}

In this paper, we propose a new method sSSR-SS for DOA estimation in the framework of sparse signal recovery with complex data. sSSR-SS takes source signal subspace as the measurement domain and exploits the parallel alliance of the basis of this signal subspace to reduce the computational cost and improve the robustness to noise. Based on the alliance, we design an optimization of sparse signal recovery for DOA estimation, reformulate it into an SOCP form, and solve it using the interior point method. We compare the performance of sSSR-SS with the classical $\ell_{1}$-SVD, OGSBISVD, and MUSIC in the numerical simulations and real data tests. The results demonstrate the effectiveness of our sSSR-SS, including no sensitivity to the assumed number of the sources, the number of resolvable sources at most, the computational complexity, and the practicability for practical application. Moreover, the experiment results verified the increased resolution and improved accuracy of sSSR-SS, especially the robustness to noise.

\section{Conflict of Interests}

The authors declare that there is no conflict of interests regarding the publication of this paper. 


\section{Acknowledgments}

This research was supported by the National Natural Science Foundation of China under Grants (nos. 61205190, 61201332, 61201337, and 61271437) and Basic Research Plan of NUDT (nos. JC13-02-03 and JC12-02-05).

\section{References}

[1] E. J. Candès, J. Romberg, and T. Tao, "Robust uncertainty principles: exact signal reconstruction from highly incomplete frequency information," IEEE Transactions on Information Theory, vol. 52, no. 2, pp. 489-509, 2006.

[2] D. L. Donoho, "Compressed sensing," IEEE Transactions on Information Theory, vol. 52, no. 4, pp. 1289-1306, 2006.

[3] R. G. Baraniuk, "Compressive sensing," IEEE Signal Processing Magazine, vol. 24, no. 4, pp. 118-124, 2007.

[4] M. Elad, Sparse and Redundant Representations: From Theory to Applications in Signal and Image Processing, Springer, New York, NY, USA, 2010.

[5] U. Gamper, P. Boesiger, and S. Kozerke, "Compressed sensing in dynamic MRI," Magnetic Resonance in Medicine, vol. 59, no. 2, pp. 365-373, 2008.

[6] J. Trzasko and A. Manduca, "Highly undersampled magnetic resonance image reconstruction via homotopic 10-minimization," IEEE Transactions on Medical Imaging, vol. 28, no. 1, pp. 106-121, 2009.

[7] Z. L. Zhang, T. P. Jung, S. Makeig, and B. D. Rao, "Compressed sensing of EEG for wireless telemonitoring with low energy consumption and inexpensive hardware," IEEE Transactions on Biomedical Engineering, vol. 60, no. 1, pp. 221-224, 2013.

[8] Z. L. Zhang, T. P. Jung, S. Makeig, and B. D. Rao, "Compressed sensing for energy-efficient wireless telemonitoring of noninvasive fetal ECG via block sparse Bayesian learning," IEEE Transactions on Biomedical Engineering, vol. 60, no. 2, pp. 300309, 2013.

[9] J. Haupt, W. U. Bajwa, M. Rabbat, and R. Nowak, "Compressed sensing for networked data: a different approach to decentralized compression," IEEE Signal Processing Magazine, vol. 25, no. 2, pp. 92-101, 2008.

[10] M. A. Davenport, C. Hegde, M. F. Duarte, and R. G. Baraniuk, "Joint manifolds for data fusion," IEEE Transactions on Image Processing, vol. 19, no. 10, pp. 2580-2594, 2010.

[11] X. M. Zhao, L. X. Zhang, P. Shi, and H. R. Karimi, "Robust control of continuous-time systems with state-dependent uncertainties and its application to electronic circuits," IEEE Transactions on Industrial Electronics, vol. 61, no. 8, pp. 4161-4170, 2014.

[12] X. M. Zhao, H. Liu, and J. F. Zhang, "Multiple-mode observer design for a class of switched linear systems linear systems," IEEE Transactions on Automation Science and Engineering, 2013.

[13] J. Chen and X. Huo, "Theoretical results on sparse representations of multiple-measurement vectors," IEEE Transactions on Signal Processing, vol. 54, no. 12, pp. 4634-4643, 2006.

[14] M. Mishali and Y. C. Eldar, "Reduce and boost: recovering arbitrary sets of jointly sparse vectors," IEEE Transactions on Signal Processing, vol. 56, no. 10, pp. 4692-4702, 2008.

[15] E. van den Berg and M. P. Friedlander, "Theoretical and empirical results for recovery from multiple measurements," IEEE Transactions on Information Theory, vol. 56, no. 5, pp. 25162527, 2010.
[16] D. Malioutov, M. Çetin, and A. S. Willsky, "A sparse signal reconstruction perspective for source localization with sensor arrays," IEEE Transactions on Signal Processing, vol. 53, no. 8, pp. 3010-3022, 2005.

[17] M. M. Hyder and K. Mahata, "Direction-of-arrival estimation using a mixed $\ell_{2,0}$ norm approximation," IEEE Transactions on Signal Processing, vol. 58, no. 9, pp. 4646-4655, 2010.

[18] P. Stoica, P. Babu, and J. Li, "SPICE: a sparse covariance-based estimation method for array processing," IEEE Transactions on Signal Processing, vol. 59, no. 2, pp. 629-638, 2011.

[19] J. M. Kim, O. K. Lee, and J. C. Ye, "Compressive MUSIC: revisiting the link between compressive sensing and array signal processing," IEEE Transactions on Information Theory, vol. 58, no. 1, pp. 278-301, 2012.

[20] W.-J. Zeng, H. C. So, and L. Huang, " $\ell_{p}$-MUSIC: robust direction-of-arrival estimator for impulsive noise environments," IEEE Transactions on Signal Processing, vol. 61, no. 17, pp. 42964308, 2013.

[21] N. Hu, Z. F. Ye, X. Xu, and M. Bao, "DOA estimation for sparse array via sparse signal reconstruction," IEEE Transactions on Aerospace and Electronic Systems, vol. 49, no. 2, pp. 760-772, 2013.

[22] E. T. Northardt, I. Bilik, and Y. I. Abramovich, "Spatial compressive sensing for direction-of-arrival estimation with bias mitigation via expected likelihood," IEEE Transactions on Signal Proc essing, vol. 61, no. 5, pp. 1183-1195, 2013.

[23] J. M. Zheng and M. Kaveh, "Sparse spatial spectral estimation: a covariance fitting algorithm, performance and regularization," IEEE Transactions on Signal Processing, vol. 61, no. 11, pp. 27672777, 2013.

[24] Z. M. Liu, Z. T. Huang, and Y. Y. Zhou, "An efficient maximum likelihood method for direction-of-arrival estimation via sparse Bayesian learning," IEEE Transactions on Wireless Communications, vol. 11, no. 10, pp. 3607-3617, 2012.

[25] Z. Yang, L. Xie, and C. Zhang, "Off-grid direction of arrival estimation using sparse Bayesian inference," IEEE Transactions on Signal Processing, vol. 61, no. 1, pp. 38-43, 2013.

[26] M. Carlin, P. Rocca, G. Oliveri, F. Viani, and A. Massa, "Directions-of-arrival estimation through Bayesian compressive sensing strategies," IEEE Transactions on Antennas and Propagation, vol. 61, no. 7, pp. 3828-3838, 2013.

[27] Z.-M. Liu and Y.-Y. Zhou, "A unified framework and sparse Bayesian perspective for direction-of-arrival estimation in the presence of array imperfections," IEEE Transactions on Signal Processing, vol. 61, no. 15, pp. 3786-3798, 2013.

[28] R. O. Schmidt, "Multiple emitter location and signal parameter estimation," IEEE Transactions on Antennas and Propagation, vol. AP-34, no. 3, pp. 276-280, 1986.

[29] F. Alizadeh and D. Goldfarb, "Second-order cone programming," Mathematical Programming. A Publication of the Mathematical Programming Society, vol. 95, no. 1, pp. 3-51, 2003.

[30] M. S. Lobo, L. Vandenberghe, S. Boyd, and H. Lebret, "Applications of second-order cone programming," Linear Algebra and its Applications, vol. 284, no. 1-3, pp. 193-228, 1998.

[31] J. S. Sturm, Using SeDuMi 1. 02, a Matlab toolbox for optimization over symmetric cones (updated for version 1. 05), Department of Econometrics, Tilburg University, Tilburg, Netherlands, http://sedumi.ie.lehigh.edu/wp-content/sedumidownloads/usrguide.ps. 


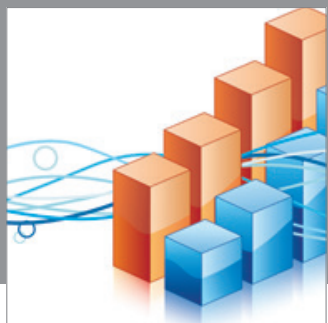

Advances in

Operations Research

mansans

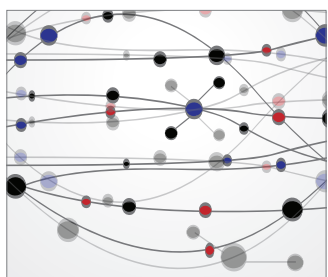

The Scientific World Journal
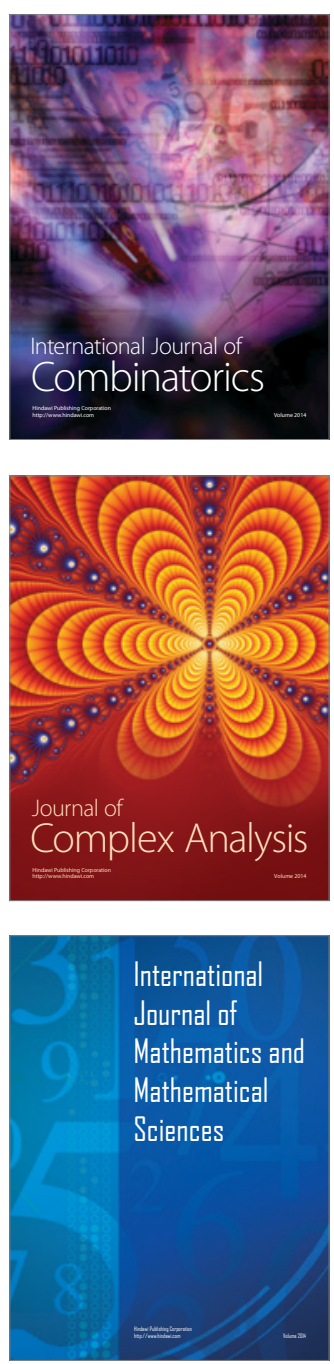
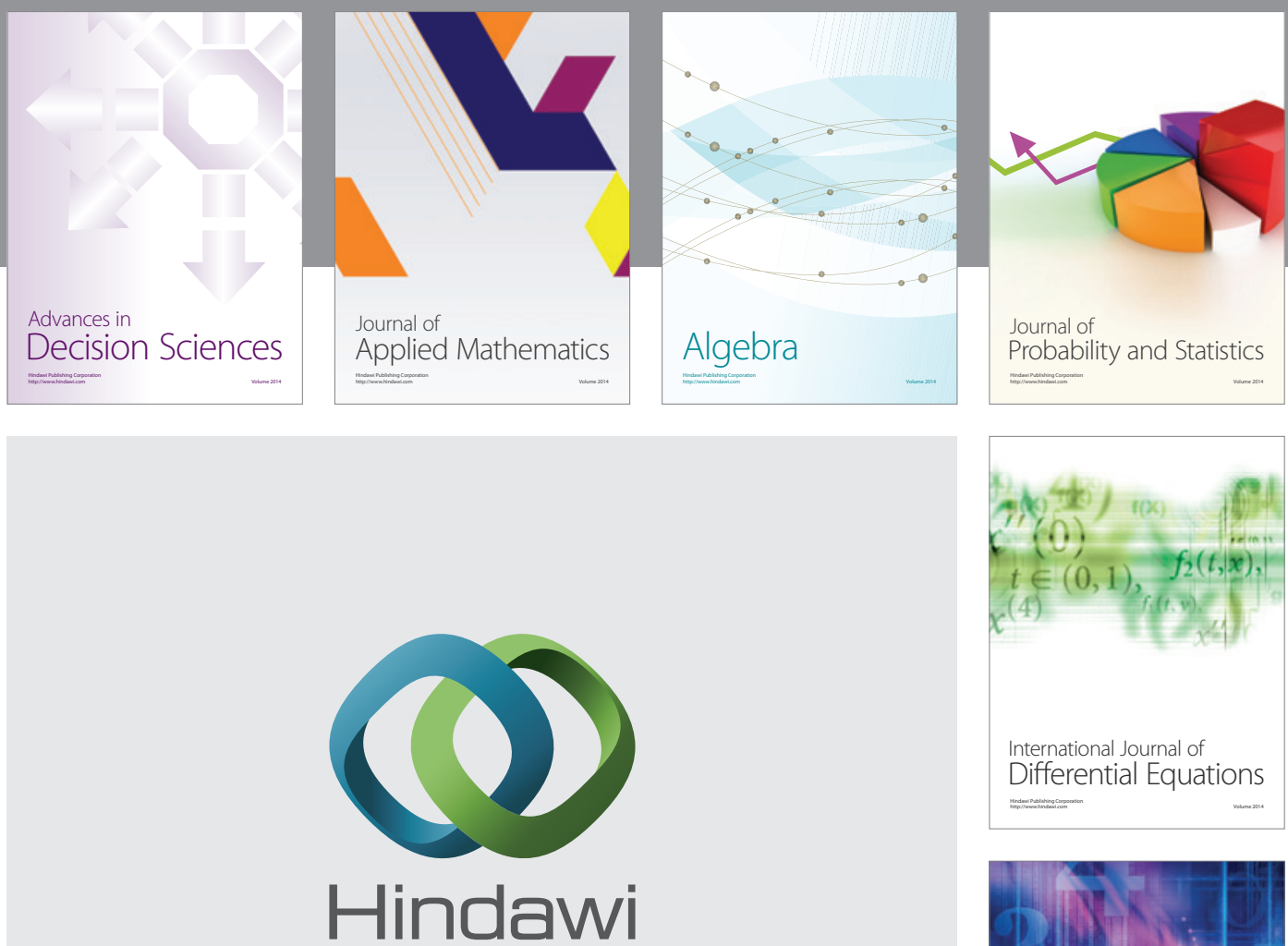

Submit your manuscripts at http://www.hindawi.com


Journal of

Function Spaces

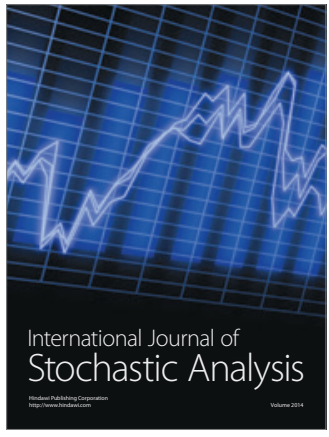

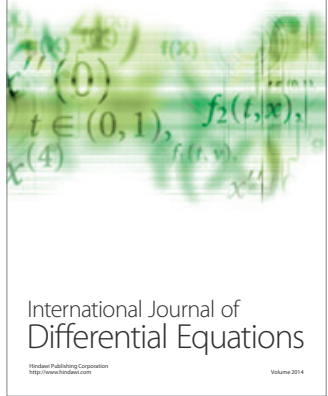
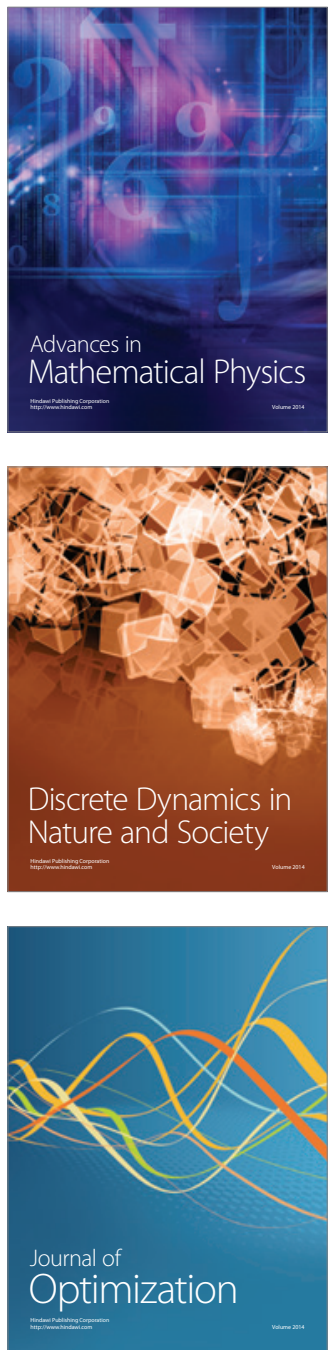